\title{
Submarine Attacking Surface Ship Combat Based on Petri Net Modeling Method
}

\author{
Hanguo Peng ${ }^{1, a}$ and Yangzhao Yang ${ }^{1, b}$ \\ ${ }^{1}$ Naval Submarine Academic of simulator training center, Dalian, 116025, China \\ azyynavy@163.com, b285892287@qq.com
}

Keywords: CGF; Warship antisubmarine simulation; Behavior modeling; Petri nets; Tactical rules

\begin{abstract}
Accurate effectively to submarine CGF modeling is to ensure that ships latent simulation submarine force in the basis of physical authenticity and intelligent behavior. In submarine CGF model based on the generic framework for submarine CGF physical modeling and behavioral modeling has carried on the analysis and research, put forward in the submarine CGF behavior model based on Petri net tactical rules introduced in front predicate formula of behavior modeling method, improves the accurate description in the battlefield of submarine CGF behavior ability. On STAGE simulation environment verification results show that the method is reasonable and effective.
\end{abstract}

\section{Introduction}

Computer Generated Forces (CGF Computer Generated Forces) refers to the battlefield environment Generated by Computer simulation and control of simulation entity, the entity can automatically respond to events in battlefield environment simulation and state. CGF has become an important part of distributed interactive simulation system.

CGF modeling technology is in recent years, along with the development of advanced distributed simulation and artificial intelligence technology and the rise of a new field of military simulation modeling, the research content including environmental modeling, physical modeling and behavioral modeling, involving multiple disciplines of crossover and integration, simulation and modeling is a very important research direction in CGF behavior model is the key to construct CGF system, also is the core of CGF modeling and the difficulty.

The main methods are: CGF behavior modeling of rule-based reasoning, neural network, Bayesian network, and the finite state machine, etc. These methods in the description of the operational simulation of complex against behavior, knowledge representation problem of autonomous decision-making behavior too simplistic and other shortcomings. Authenticity is still lack of CGF behavior now needs to be improved in the field of military simulation is a problem.

In this paper, the ship to potential confrontation simulation system of submarine CGF modeling method of physical and behavioral analysis and research, in using Petri nets for submarine CGF behavior modeling, on the basis of the introduction of prepositional predicate formula for changes of additional condition such as submarine tactics and weapons used rules, improve the accurate description conforms to the battlefield of submarine CGF behavior ability.

\section{Submarine CGF Model Framework}

Submarine CGF is the ship on the submarine combat system simulation of submarine forces, is the key to accurate and reasonable physical modeling and behavioral modeling, to reflect against submarine force in the process of physical authenticity and intelligent behavior. CGF modeling mainly includes the physical modeling and behavioral modeling, also should consider the elements of environment model. Physical model describes the simulation prototype characteristics and regularity of objective existence, behavioral model describes the simulation prototype according to the external environment and their own situation to make a decision and take action to intelligent behavior. Behavioral model based on physical model, through physical model for battlefield perception and intelligence, command, on the basis of decision-making are generated from the 
reasoning, and implement decisions through the physical model, the produce effect, apply to the battlefield environment. Natural environment and environment model (combat troops, environment) is a simulation of forces entities run outside of the bottom support.

The main part of the Submarine CGF generic framework are as follows: physical model, autonomous behavior produce module and operator control module. In the process of system simulation scenarios, submarine CGF external information through the network interface (environment information, the situation of the enemy), for situation assessment, decision-making and Action planning to provide information support, and through the autonomous behavior produce module tactics of knowledge base, operational rules in rule base - matching rules, make tactical decisions and implementation, form the motion planning sequence (COA, Course of Action). For each specific platform for the motion planning, a dynamic model and weapon system model to calculate the corresponding parameters, and in accordance with the rules of the corresponding tactics and weapons used rule execution to show such as fire platform acceleration, steering, weapons, etc.

\section{The Submarine CGF Physical Modeling}

The submarine CGF physical modeling focused on the description of physical properties, generally divided into static characteristics and dynamic characteristics of the two. Static characteristics of submarine size, displacement, moment of inertia, a physical model parameters such as the inherent nature of weapons and equipment. Dynamic characteristics including submarine underwater maneuvering rules, the passive sonar of ship target detection found that regular physical model of dynamic simulation, etc.

Combat simulation system, in the ship and submarine ships the description of the features include: basic information (size, weight, maximum depth, maximum receiving depth, maximum speed, etc.), motor model parameters, dynamic self-force (oil, battery power), noise (radiation, reflection noise), carrying weapons and equipment of the tactical technical performance, etc.

The description of the 2.2 submarine dynamic characteristics

The dynamic characteristics of a submarine CGF described mainly through the establishment of submarines, sensor model, dynamic model of weapon system performance model, etc. These aspects of modeling method is relatively mature, this article mainly discusses the dynamic model of submarine platform, sensor described.

Submarines without trim constant depth motion equation of motion:

$\Delta \mathrm{P}+\mathrm{Z} *+\mathrm{Z} \delta \mathrm{b}+\mathrm{Z} \delta \mathrm{s}=0$

$\Delta \mathrm{M}+\mathrm{M}^{*}+\mathrm{M} \delta \mathrm{b}+\mathrm{M} \delta \mathrm{s}=0$

Among them: $\Delta \mathrm{P}$ — poor buoyancy, $\Delta \mathrm{M}$ - torque, $\mathrm{Z} *, \mathrm{M} *$ —-due to the asymmetry of the boat body up and down hydrodynamic (moment), Z $Z \delta b 、 M \delta b-$ wai shell rudder force (torque), $\mathrm{Z} \delta \mathrm{s} 、 \mathrm{M} \delta \mathrm{s}$ - - stern rudder force (torque), $\delta \mathrm{b}$ - - wai shell rudder and rudder Angle, $\delta \mathrm{s}$ - - stern rudder and rudder Angle.

Submarine detected by active sonar's ships in the process of active sonar equation described as:

$\mathrm{SL}-2 \mathrm{TL}+\mathrm{TS}-\mathrm{NL}+\mathrm{GL}-\mathrm{DT}=0$

Passive sonar equation is described as:

SL-TL=NL-DI+DT

Among them: SL-—emission source level, TLintensity, NL- - its noise level, GL - its processing gain, DT - detection threshold.

\section{The Submarine CGF Behavior Modeling}

The Submarine CGF Behavior Model Based on Petri Net. Combat simulation system, in the ship and submarine CGF behavior modeling task is in the computer simulation model is used to simulate the submarine combat behavior. For submarine combat CGF behavior for more complex 
discrete event and the occurrence of the event has many characteristics, such as parallel, asynchronous and random, based on the Petri nets for submarine CGF behavior modeling.

In the standard of Petri nets graphical representation (figure 3), on behalf of the library circle, rectangle represents change, have said to the arc from library to change the input and output from changes to the library. In the process of simulation entity behavior modeling, generally use the concept of events and activities. Mapped to a Petri net, library means change the representative action. A change has a certain number of input and output, respectively, on behalf of the submarine CGF operational activities of the trigger events and termination. The tag library (Token) notation denotes the simulation entity activity using resources, or data.

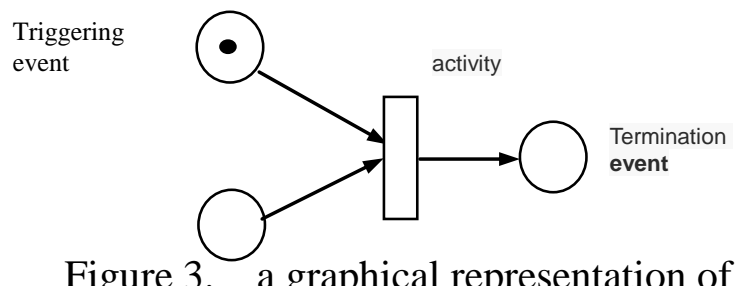

Figure 3. a graphical representation of the Petri net model

Submarine CGF behavior (navigation, exploration, torpedo attack, etc.) are mostly discrete events, these events need to meet certain conditions or as part of the "accumulation" can trigger, for example, the detection behavior is submarine sonar detection target information of the combination of a series of actions, until the submarine found target, its detection behavior will terminate, into the tracking behavior. So, in the submarine CGF behavior modeling of a very important problem is through a concrete analysis of submarine tactical action clear submarine tactical action categories, tactical action triggering event, tactical action termination events, etc. Based on Petri net work flow analysis, in the discrete different categories of submarine tactical action can use the changes of the Petri net is described, and the tactical action can trigger events and end use Petri net library are described, so that we can use composed of multiple changes and the library of Petri nets to describe various tactical submarine CGF behavior.

The Submarine CGF Basic Behavior of the Object. Submarine CGF basic behavior of the object is to point to by the analysis of real world submarine combat process, concludes that can represent the submarine combat missions in several classes of the same or similar nature. On the reasonable combination of submarine CGF behavior basic object and trade-offs, and can accurately describe the ship hidden submarine CGF simulation system of action.

Submarine CGF basic behavior of the object is divided into four categories: motor behavior object, search behavior object, the object of attack and defense behavior object. Accordingly to establish the basic object of behavior of the Petri net model can be detailed and clearly describe the submarine CGF behavior.

Prepositional Predicate Formula Based on Tactical Rules. Submarine combat CGF behavior has the characteristics of parallel, asynchronous, in the process of the whole ship to dive against submarine CGF entity in the next moment need to take action according to the battlefield and the tactical rules to determine, using only the basic Petri nets to describe this operational process is not enough, need to give each change happen attach corresponding conditions, these conditions must follow tactics and weapons used rules. In particular battlefield environment, only additional conditions up to the changes of the tactics and weapons used rules could be implemented.

According to the definition and change of Petri net conditions, this paper introduces a prepositional predicate formula based on tactical rules.

Define prepositional predicate formula: $C_{b}$ front predicate formula, $t_{i \in T}$ called change, if and only if:

(1) $C_{b}$ is one of the time, the network state of the system and network system are identified as the predicate formulas of argument; 
(2) $C_{b}$ is the necessary condition to change;

(3) When $C_{b}$ is true, and there is no conflict, change ${ }^{t_{i}}$, when there is conflict, conflict strategy should change occurs depends on the change.

There are four kinds of conflict resolution change strategy:

(1) Will be introduced to the priority change trigger event, the high priority trigger event triggers the corresponding change;

(2) And tactical rules associated will change, meet the changes of tactical rules implemented;

(3) The triggering event of introducing temporal relations change, instantaneous triggering event than triggering events with the time parameter of high priority, the changes of the time parameter small than time parameter change implementation of priority;

(4) have the same implementations of priority change according to the probability to determine which changes to implement.

Attack Submarine CGF Behavior Petri Net Model. To attack submarine CGF behavior model as an example, its predicate formula Petri net model as shown in figure 4. Libraries in this picture represents submarine attacks in various events, change represents a submarine attack submarine tactical activities. Petri net library, change and prepositional predicate formula of distribution such as shown in table 1 and table 2 and table 3.

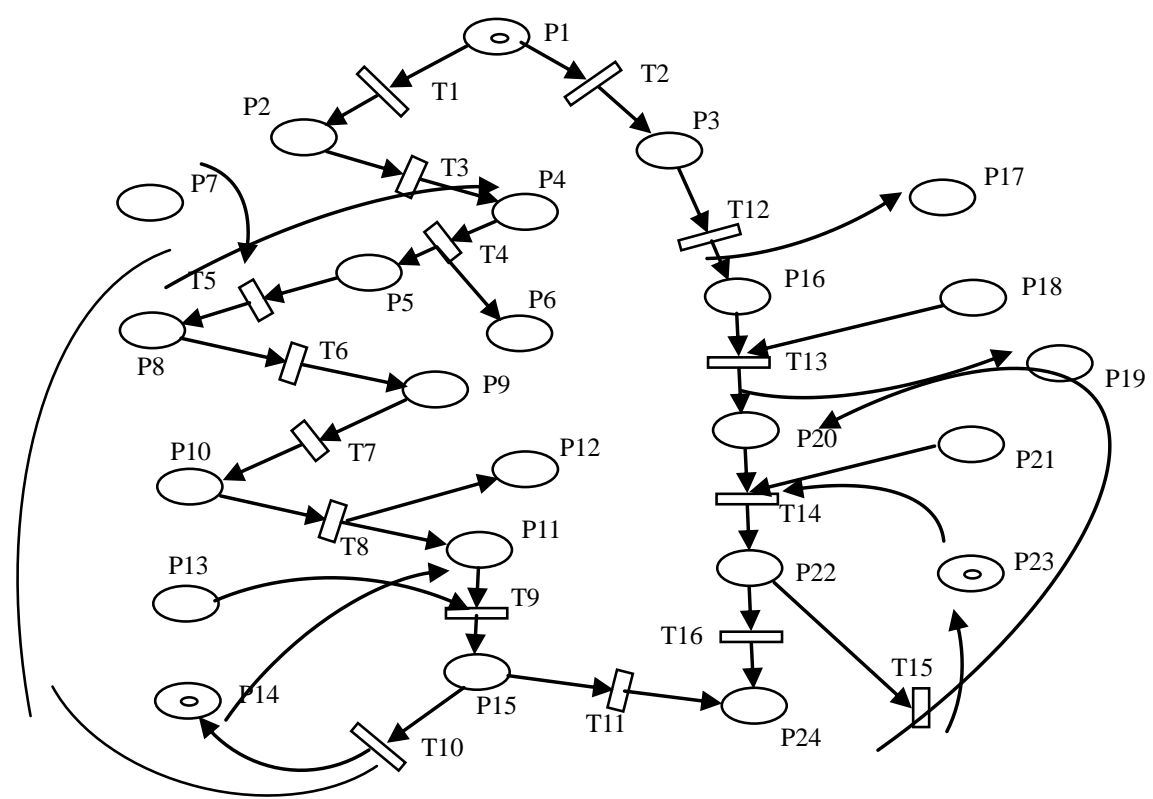

Figure 4. Petri nets model of submarine attacking CGF 
Table 1 attack of Petri net library

\begin{tabular}{|c|c|c|c|}
\hline location & instructions & location & instructions \\
\hline $\mathrm{P} 1$ & Began to attack the target effectively & $\mathrm{P} 2$ & Start the torpedo attack \\
\hline P3 & Start missile attacks & $\mathrm{P} 4$ & Begin to choose target \\
\hline P5 & Began to break through the alert screen & P6 & $\begin{array}{l}\text { Apply for breakthrough alert } \\
\text { screen mobile tag }\end{array}$ \\
\hline P7 & Break through the motor end tag & P8 & Begin to choose types of torpedo \\
\hline P9 & Begin to choose shooting method & $\mathrm{P} 10$ & Begin to choose shooting form \\
\hline P11 & Began to occupy the fire & P12 & $\begin{array}{l}\text { Apply for motor to occupy the fire } \\
\text { position }\end{array}$ \\
\hline P13 & $\begin{array}{l}\text { Apply for motor to occupy the fire } \\
\text { position }\end{array}$ & P14 & The torpedo available tags \\
\hline P15 & Started to launch torpedo & P16 & Occupy the attack to array \\
\hline P17 & $\begin{array}{l}\text { Apply for occupation attack on a motor } \\
\qquad \operatorname{tags}\end{array}$ & P18 & $\begin{array}{l}\text { Occupation of the attack on a } \\
\text { motor end tag }\end{array}$ \\
\hline P19 & $\begin{array}{l}\text { Apply for occupation of transmitting } \\
\text { array a motor tags }\end{array}$ & $\mathrm{P} 20$ & $\begin{array}{l}\text { Began to occupy the transmitting } \\
\text { array }\end{array}$ \\
\hline $\mathrm{P} 21$ & $\begin{array}{c}\text { Occupy the transmitting array a motor } \\
\text { end tag }\end{array}$ & $\mathrm{P} 22$ & Start a missile \\
\hline $\mathrm{P} 23$ & Missile available tags & $\mathrm{P} 24$ & End of the attack \\
\hline
\end{tabular}

Table 2 The changes of aggression Petri net

\begin{tabular}{|c|c|c|c|}
\hline location & instructions & location & instructions \\
\hline T1 & Decided to use torpedo weapon attack & $\mathrm{T} 2$ & Decided to use a missile attack \\
\hline $\mathrm{T} 3$ & Choose a target & $\mathrm{T} 4$ & Breakthrough alert screen \\
\hline T5 & $\begin{array}{l}\text { Breakthrough alert screen motor end } \\
\text { tag non-ferrous effectively }\end{array}$ & T6 & Determine the types of torpedo \\
\hline $\mathrm{T} 7$ & $\begin{array}{c}\text { Finished shooting method is } \\
\text { determined }\end{array}$ & T8 & $\begin{array}{l}\text { Determine the shooting finished } \\
\text { form }\end{array}$ \\
\hline T9 & $\begin{array}{l}\text { Motor end tag to occupy the fire } \\
\text { position non-ferrous effectively }\end{array}$ & $\mathrm{T} 10$ & $\begin{array}{c}\text { Decided to continue to attack and } \\
\text { torpedo available }\end{array}$ \\
\hline $\begin{array}{r}\mathrm{T} 11 、 \\
\mathrm{~T} 16\end{array}$ & End of the attack & $\mathrm{T} 12$ & $\begin{array}{l}\text { Choose to attack start fighting } \\
\text { positions }\end{array}$ \\
\hline $\mathrm{T} 13$ & $\begin{array}{l}\text { Occupation of the attack on a motor to } \\
\text { complete colored markers effectively }\end{array}$ & $\mathrm{T} 14$ & $\begin{array}{l}\text { Occupy the transmitting array a } \\
\text { motor end tag non-ferrous } \\
\text { effectively }\end{array}$ \\
\hline $\mathrm{T} 15$ & $\begin{array}{l}\text { Decided to continue to attack and } \\
\text { missile is available }\end{array}$ & & \\
\hline
\end{tabular}


Table 3 attacks prior predicate description of Petri nets

\begin{tabular}{|c|c|c|c|}
\hline location & instructions & location & instructions \\
\hline $\mathrm{C} 1$ & The rules of choosing weapons & $\mathrm{C} 2$ & The rules of choosing weapons \\
\hline $\mathrm{C} 3$ & Choose target rules & $\mathrm{C} 4$ & $\begin{array}{l}\text { Judge breakthrough act alert } \\
\text { rules }\end{array}$ \\
\hline $\mathrm{C} 5$ & $\begin{array}{l}\text { Breakthrough alert screen motor } \\
\text { end tag non-ferrous effectively }\end{array}$ & C6 & $\begin{array}{c}\text { Determine the torpedo type } \\
\text { rules }\end{array}$ \\
\hline $\mathrm{C} 7$ & $\begin{array}{c}\text { Rules of the shooting method is } \\
\text { determined }\end{array}$ & $\mathrm{C} 8$ & $\begin{array}{c}\text { Determine the shooting finished } \\
\text { form }\end{array}$ \\
\hline C9 & Motor to occupy the fire position & $\mathrm{C} 10$ & The rules of decided to attack \\
\hline C11、C16 & Attack judgment rules & $\mathrm{C} 12$ & $\begin{array}{l}\text { Choose to attack the rules of the } \\
\text { fighting positions }\end{array}$ \\
\hline C13 & $\begin{array}{l}\text { Occupation of the attack on a } \\
\text { motor to complete colored } \\
\text { markers effectively }\end{array}$ & $\mathrm{C} 14$ & $\begin{array}{c}\text { Occupy the transmitting array a } \\
\text { motor end tag non-ferrous } \\
\text { effectively }\end{array}$ \\
\hline $\mathrm{C} 15$ & Decided to attack the rules & & \\
\hline
\end{tabular}

Submarine attacking CGF behavior processes of Petri nets model of explanation: if started attacking target effective, T1, T2 competition change, change, conform to the rules of tactical implementation of change. If according to $\mathrm{C} 1$ decide use torpedo weapon attack target, $\mathrm{T} 1$ to implement the change, the submarine entered the state began to torpedo attack the P2; If according to $\mathrm{C} 1$ determine using missile target, $\mathrm{T} 2$ to implement the change, into a state submarine missile attack P3.

T3, if use torpedo weapon, change according to C3 choose targets, T4 implementation choice after the completion of change, on the one hand, send out application breakthrough alert screen mobile tag, on the one hand into the break through the state of alert screen P5. According to the C4 determine whether breakthrough alert screen, if it is true, change T5, tag into the P8, depending on the type of C6 select torpedoes, change T6, then according to the C7 determine whether submarines corner shooting, choose according to $\mathrm{C} 8$ determine what shooting form. Change $\mathrm{T} 8$ motor tag to send application occupation the fire position, at the same time make the tag into the state P11 to occupy the fire position. If is to occupy the fire position, and the torpedo available T9 to implement the change, the submarine began to launch torpedo, after the launch, according to the needs of C10 if continue to use the torpedo attack, T10 to implement the change, send the torpedo available tags, and return to select the target state P4. If end of the attack, the attack end tag changes T11 sent. Submarine CGF behavior modeling on the use of missile weapons slightly.

\section{Conclusions}

Submarine CGF model to improve ship combat simulation accuracy and scalability of the research on anti-submarine warfare is of great significance. In this paper, the submarine CGF modeling method was studied, the physical model of submarine CGF construction has carried on the brief narration, then in view of the current behavior modeling difficulty and the bottleneck problem, the traditional extended Petri net model, will lead the tactical predicate associated with the change of Petri nets, and based on prepositional predicate Petri net submarine CGF behavior modeling method, this method can satisfy the description ships latent in combat simulation has the characteristics of parallel, asynchronous and random events, can satisfy the submarine CGF intelligent behavior generated, developed and applied in the corresponding simulation system, the results are applied to the other forces of CGF modeling. 


\section{References}

[1] Liwei L, Rongshuang F. Simulated annealing algorithm in solving frequency assignment problem[C]//Advanced Computer Theory and Engineering (ICACTE), 2010 3rd International Conference on. IEEE, 2010, 1: V1-361-V1-364.

[2] Castelino D J, Hurley S, Stephens N M. A tabu search algorithm for frequency assignment [J]. Annals of Operations Research, 1996, 63(2): 301-319.

[3] Alabau M, Idoumghar L, Schott R. New hybrid genetic algorithms for the frequency assignment problem [J]. Broadcasting, IEEE Transactions on, 2002, 48(1): 27-34.

[4] Radiom, S., Aliakbarian, H., etc., A Simple Real-Coded Compact Genetic Algorithm and its Application to Antenna Optimization, Microwave conference, APMC 2007.

[5] Francisco Herrera and Manuel Lozano. Gradual Distributed Real-Coded Genetic Algorithms [J]. IEEE transactions on evolutionary computation, 2000, 4(1):43-62.

[6] Díógenes Marcano and Filinto Durán. Synthesis of [7] Antenna Arrays Using Genetic Algorithms [J]. IEEE Antennas and Propagation Magazine, 42(3):12-20.

[7] Wen-Pin Liao, Fu-Lai Chu. Array pattern synthesis with null steering using genetic algorithm by controlling only the current amplitudes [J]. INT. J. ELECTRONICS, 1999, 86(4):445-457.

[8] Wen-Pin Liao, Fu-Lai Chu Application of Genetic Algorithms to Phase-Only Null Steering of Linear Arrays [J] Electromagnetics, 1997, 17:171-183.

[9] Fred Aust in, Michael lew is. Automated Maneuvering Decision for Air-to-Air Combat[R].AIAA-87-2393:659-669 